\title{
PULSARS, THEIR EVOLUTION, WINDS AND RADIATION
}

\author{
W. KUNDT \\ Institut für Astrophysik der Universität Bonn
}

\begin{abstract}
Controversial interpretations of the functioning and evolution of (radio) pulsars are discussed and confronted with the constraints. It is argued that (i) polarized $e^{ \pm}$bunches are ejected from near the polar caps at high Lorentz factors $\gamma \gtrsim 10^{2}$, that (ii) coherent radio pulses are emitted by them during transit to the outer magnetosphere via curvature radiation, (iii) in the shape of (transverse) fan beams, that (iv) there is no indication of magnetic field decay (or buildup), and that (v) pulsars die either by alignment or by interstellar quenching.

The so-called "millisecond" pulsars (outside globular clusters) are likely to be an abundant, young population of fast-born pulsars.
\end{abstract}

\section{Pulsar wind dynamics}

Pulsar winds have been mapped for the Crab and Vela pulsars, the eclipsing millisecond pulsar $1957+20$ and, probably, the jet sources Cyg X-3, SS 433, A 1850-08 and others. They are thought to consist of relativistic pair plasma, because relativistic electrons are required for the observed pulses, and because protons (at the same speed, to keep electric currents limited) would (i) exceed the available power, (ii) cause a different morphology of the Crab nebula (because they are stiffer, and do not lose their stiffness by radiation), and (iii) cannot be supplied from the surface at a sufficient rate (see below), as opposed to $\mathrm{e}^{ \pm}$pairs (produced in vacuum cascades).

For the Crab pulsar and for PSR 1957+20, the escaping electron rate $\dot{N}_{e}=I \omega|\dot{\omega}| / \gamma m_{e} c^{2}$ is large compared to the Goldreich-Julian rate $\dot{N}_{\mathrm{GJ}}=$ $\mu \omega^{2} / e c,(\mu=$ magnetic dipole moment $)$ :

$$
\dot{N}_{\mathrm{e}} / \dot{N}_{\mathrm{GJ}}=\left(\operatorname{Iec} / \mu \gamma m_{\mathrm{e}} c^{2}\right)|\dot{\omega}| / \omega \gtrsim 10^{4},
$$

whereby $\mu \gamma=10^{31.3} \mathrm{G} \mathrm{cm}^{3}$ has been inserted for PSR 1957+20; cf. Kundt (1990a), i.e., the winds are $\gtrsim 10^{4}$ times denser than if the Goldreich-Julian corotating charge density $\rho_{\mathrm{GJ}}$ were flung out radially at the speed of light. I conclude that the particles are produced in vacuum (discharges), that they are (therefore) predominantly electrons and positrons, and that they escape almost radially at $v \approx c$, guided by the corotating magnetosphere and its wave field. Models with particle infall, or screening of the strong wave, or turbulence seem inconsistent with this constraint; $c f$. Camenzind (1990).

An almost radial escape of the relativistic charges (from the polar caps to the distant wind zone) is independently indicated by the frequencydependent polarization structure of the radio pulses and subpulses, both circular and linear, all of whose details can be modelled by curvature radiation (Gil and Snakowski 1990b, Gil 1992). The required transient bunching of the radiating charges-not yet elaborated at the time of the Conference (Melrose 1981, Melrose 1992) - may be a consequence of their unsteady production in electric sparks.

Moreover, De Jager (1990) reports VHE pulses from $\mathrm{AE} \mathrm{Aqu,} \mathrm{a} \mathrm{fast-spinning}(P=33 \mathrm{~s})$, magnetized, binary white dwarf, received in phase with the optical and X-ray pulses. The power in the VHE pulses amounts to several percent of its spindown power, too large for an origin in the accretion disk. Again, near-radial escape of VHE charges is indicated for this magnetized rotator whose working scheme is expected to be similar to that of a (magnetized) neutron star.

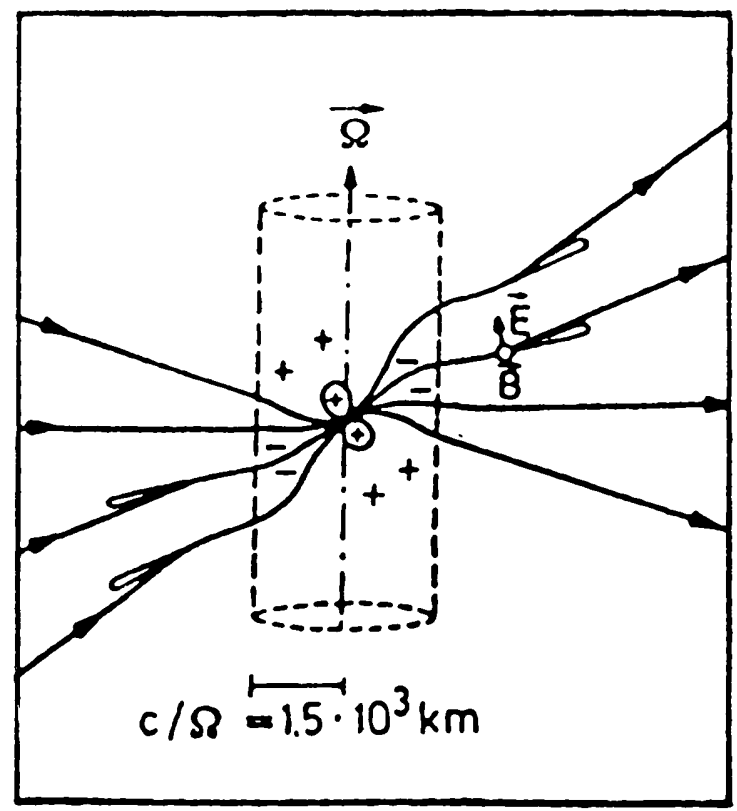

Figure 1 Schematic cross section through the magnetosphere and wind zone of an oblique rotator (of $\Omega=200 \mathrm{~s}^{-1}$ ): the charges escape more or less along the projected magnetic field lines. 


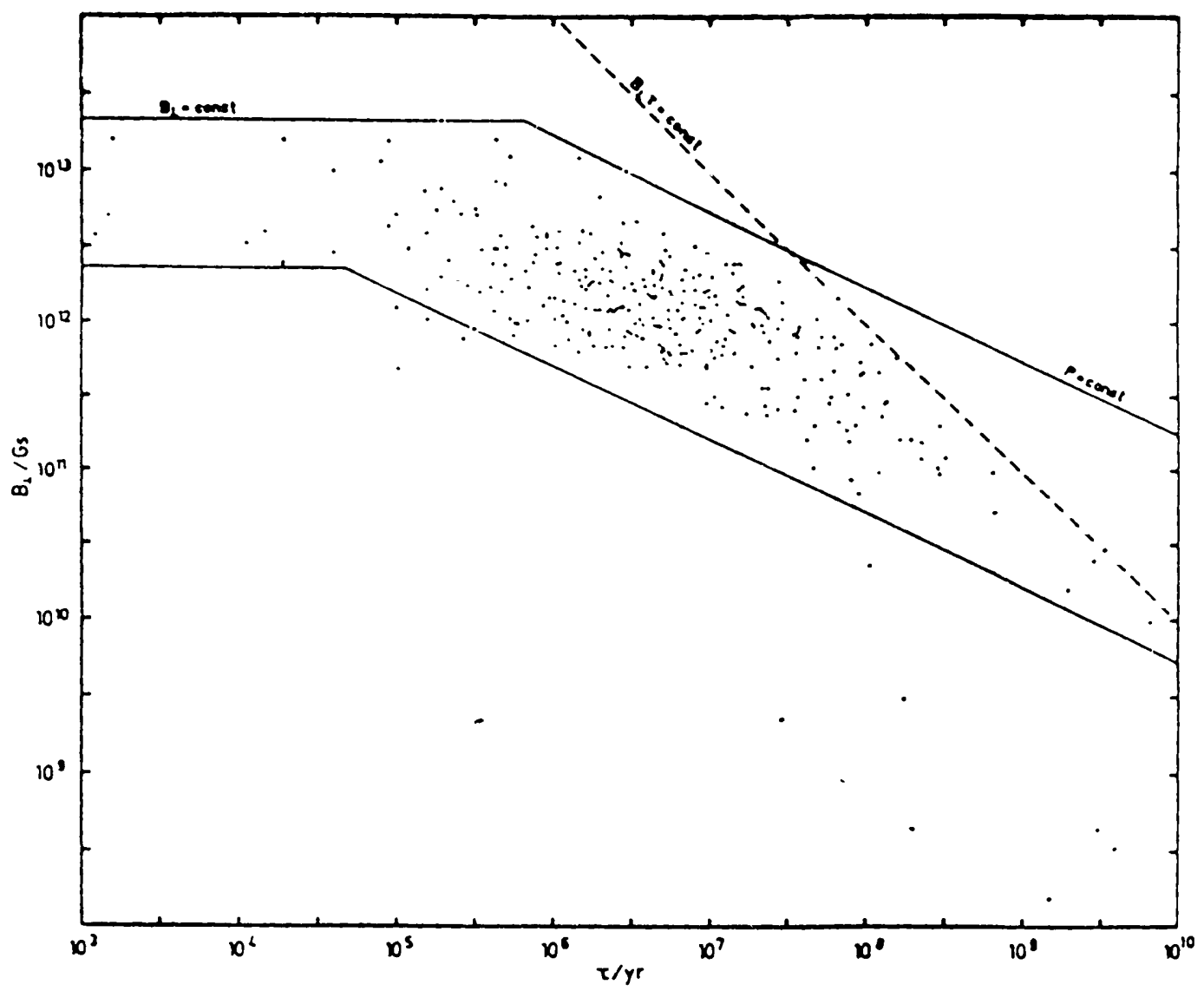

Figure 2 Values of $\left(P \dot{P} / 10^{-15} s\right)^{1 / 2}=: B_{\perp} / 10^{12} \mathrm{G}\left(I / R^{6}\right)_{9}^{1 / 2}$ versus spindown age $P / 2 \dot{P}=: \tau$ for some 300 pulsars, taken from Kundt (1990a) with a few recent (extreme) points added. The broken line $B_{\perp} \tau=10^{20} \mathrm{G} s$ is a suggestive detectability limit. The plot supports a pulsar evolution starting with $B_{\perp}=$ constant and ending with $P=$ constant, whereby pulsars die before reaching the detectability limit. The projected magnetic dipole moments of the short-period pulsars are weaker by factors of $\leq 10^{2}$ than the lower limit of the others.

\section{Charge and current density}

The Goldreich-Julian solution predicts corotating space charges in the magnetosphere whose (partial) absence would imply enormous voltages, and vacuum breakdown. As the wind is many $\left(\gtrsim 10^{4}\right)$ times denser, its net charge density tends not to deviate significantly from $\rho_{\mathrm{GJ}}$ on average. At the same time, the wind must have divergence-free electric currents: $\boldsymbol{\nabla} \cdot \boldsymbol{j}=\mathbf{0}$. If large currents were flowing, $j \gtrsim \rho_{\mathrm{GJ}} c$, they would have to close across the polar caps as Pedersen currents whose generated heat would have been detected at X-rays, (cf. figure 1 ). I conclude that $j \ll \rho_{\mathrm{GJ}} c$ holds almost everywhere, i.e. that electrons and positrons arrange for

$$
\text { both } \rho \approx \rho_{\mathrm{GJ}} \text { and } 0 \approx j=\rho_{+} v_{+}-\rho_{-} v_{-}
$$

throughout the magnetosphere (Kundt 1985). Independent reasons are the large inductive resistance, which forbids strong currents near the polar caps, and the absence of a medium that would act as a counter balance for the implied magnetic torque. Conditions (2) yield typical Lorentz factors of a few hundred of the ejected pairs.

\section{Magnetic flux decay}

Magnetic flux decay has been proposed for evolved neutron stars, but flux buildup is also occasionally considered. Historically, flux decay was suggested by the fact that pulsars turn off at $t=10^{6.7 \pm 0.3}$ $\mathrm{yr}-$ as evidenced by their kinetic ages $\left(z-z_{*}\right) / \dot{z}-$ much younger than expected on grounds of their spindown power (Kundt 1990b). Flux decay seemed also to have taken place in the (somewhat older) 'galactic-bulge' X-ray sources.

Difficulties of the flux-decay model are the facts that (i) different estimates of the decay time $\tau_{\mu}$, on the same sample, come up with different numbers (cf. figure 2); (ii) the histories of the pulse width $[W(\tau)]$ and maximal linear polarization swing $\left[\Theta^{\prime}(\varphi)\right]$ as functions of spindown age $\tau \equiv P / 2 \dot{P}$ are non-monotonic, with extrema near $\tau=10^{6.7} \mathrm{yr}$, and (iii) the braking index $n \equiv \omega \ddot{\omega} / \dot{\omega}^{2}$ correlates in magnitude with spindown age, according to $|n| \lesssim \tau / \tau_{\mathrm{c}}$, with $\tau_{\mathrm{c}} \approx 10^{2.5} \mathrm{yr}$, see figure 3. Instead of flux decay, flux alignment (with the spin) yields a more convincing interpretation; cf. Candy and Blair (1986), Kundt (1988). The alignment time scale $\tau_{\mu}$ links the true age $t$ to the spindown age $\tau$ in the 


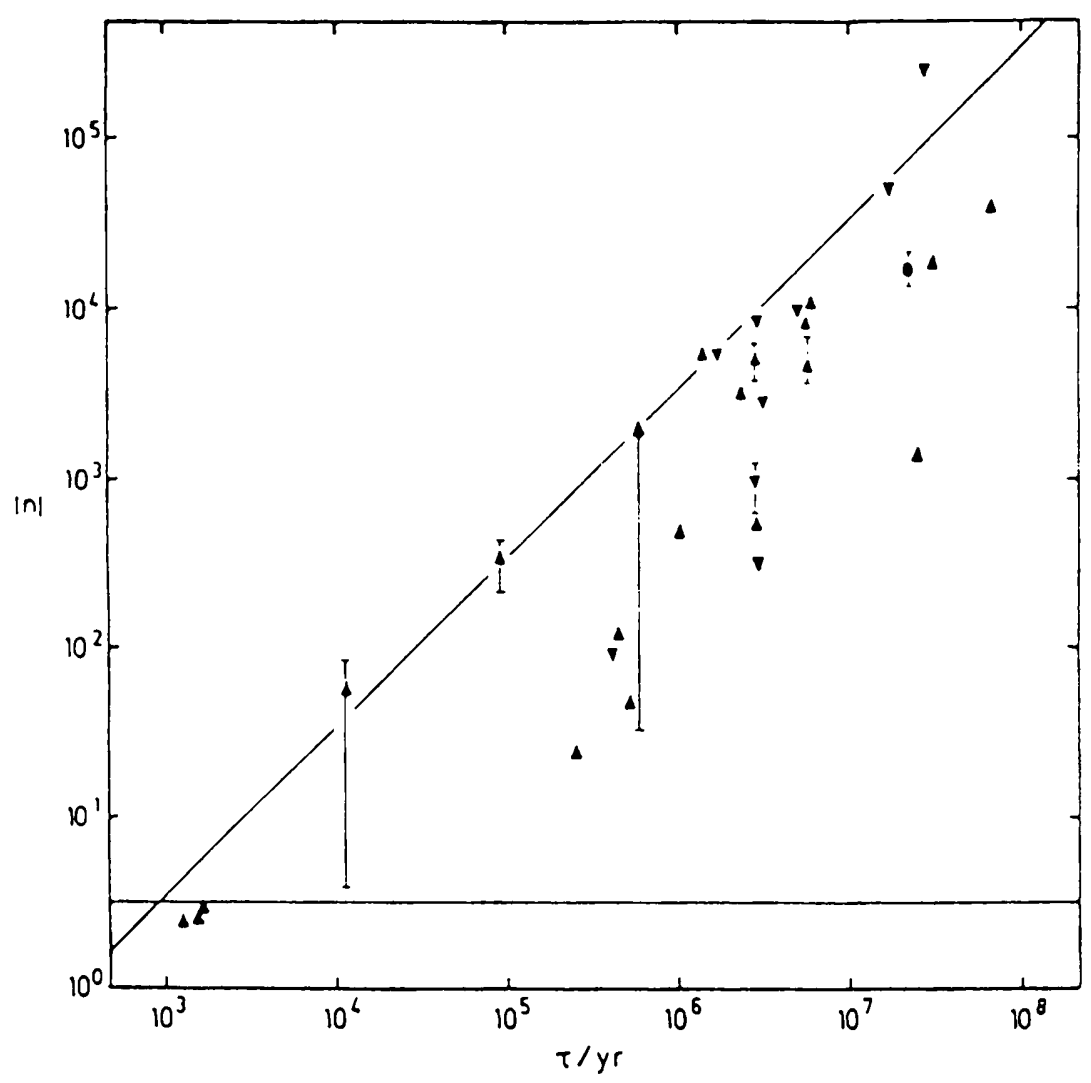

Figure 3 Magnitude of the braking index $n \equiv \omega \ddot{\omega} / \dot{\omega}^{2}$ vs. $\tau \equiv P / 2 \dot{P}$ for 30 pulsars, taken from Kundt (1988) and Shabanova (1990). Triangles pointing upward correspond to positive $n$, those pointing downward to negative $n$. The two straight lines are $|n|=\tau / 300 \mathrm{yr}$ and the dipole braking law $n=3$.

form

$$
t / \tau_{\mu}=\ln \left[1+\left(\tau-\tau_{*}\right) /\left(\tau_{\mu}-\tau_{*}\right)\right],
$$

where $\tau_{*}$ is the spindown age at birth. Alternatively, pulsar turnoff may be due to a quenching by the (relativistic) halo plasma, similar to discussions by Ostriker et al. (1970) and Kolosov et al. (1989); cf. Kundt (1981).

A further difficulty of the flux-decay model is the fact that even comparatively old binary neutron stars, like Her X-1 and 4 U 1627-67, show an $\mathrm{X}$-ray cyclotron line, corresponding to strong magnetic surface fields. Moreover, several of the lowmass X-ray binaries are jet sources and/or VHE $\gamma$-ray sources and may generate particle energies

$$
\varepsilon=e \int(E+\beta \times B) \cdot d x
$$

in excess of $10^{12} \mathrm{eV}$ near the speed-of-light cylinder (Kundt 1990a). For this they must be strongly magnetized. The same is true of the-probably extremely old- $\gamma$-ray bursters (Dermer 1990). No Xray source with a weak field has been reliably identified. Differences in temporal and spectral behavior between different $\mathrm{X}$-ray binaries may rather be due to different branching ratios of the accretion flow near the corotation cylinder: (i) down to the surface in the form of massive 'blades', (ii) down to the polar caps via 'evaporation', or (iii) out again via a magnetic slingshot (Kundt, Özel, and Ercan 1987, Horn and Kundt 1989).

\section{Beam shapes}

The radio-pulse beams have been recently claimed to be circular by Lyne and Manchester (1988), based on a reanalysis of the swing of linear polarization through the pulse. They then arrive at a beaming fraction of $f=0.14$ which is related to the birth interval $\Delta t$ of pulsars in the Galaxy through

$$
\Delta t=t f / N=10^{1.3 \pm 0.5} \mathrm{yr} f,
$$

in which $t$ is the mean pulsar age $\left(t=10^{6.7 \pm 0.3} \mathrm{yr}\right)$, and $N=10^{5.4 \pm 0.3}$ is the number of observable pulsars in the Galaxy. The inserted numbers yield a birth interval of pulsars in the Galaxy of one in $20 \mathrm{f}$ years. If every pulsar has at least one older brother (whose formation kicked it to its large scale height at birth, of $\lesssim 200 \mathrm{pc}$ ), the birth interval of neutron stars shrinks below one in $10 f$ years, embarrassingly short for the supernova rate if $f \ll 1$ (Kundt 1990b).

Obviously, the strength of the argument rests on the uncertainties in $t$ and $N$. As for $t$, there is a recent tendency (not shared by myself) to raise the mean pulsar lifetime towards $10^{7} \mathrm{yr}$. The estimate depends on kinetic-age determinations which 


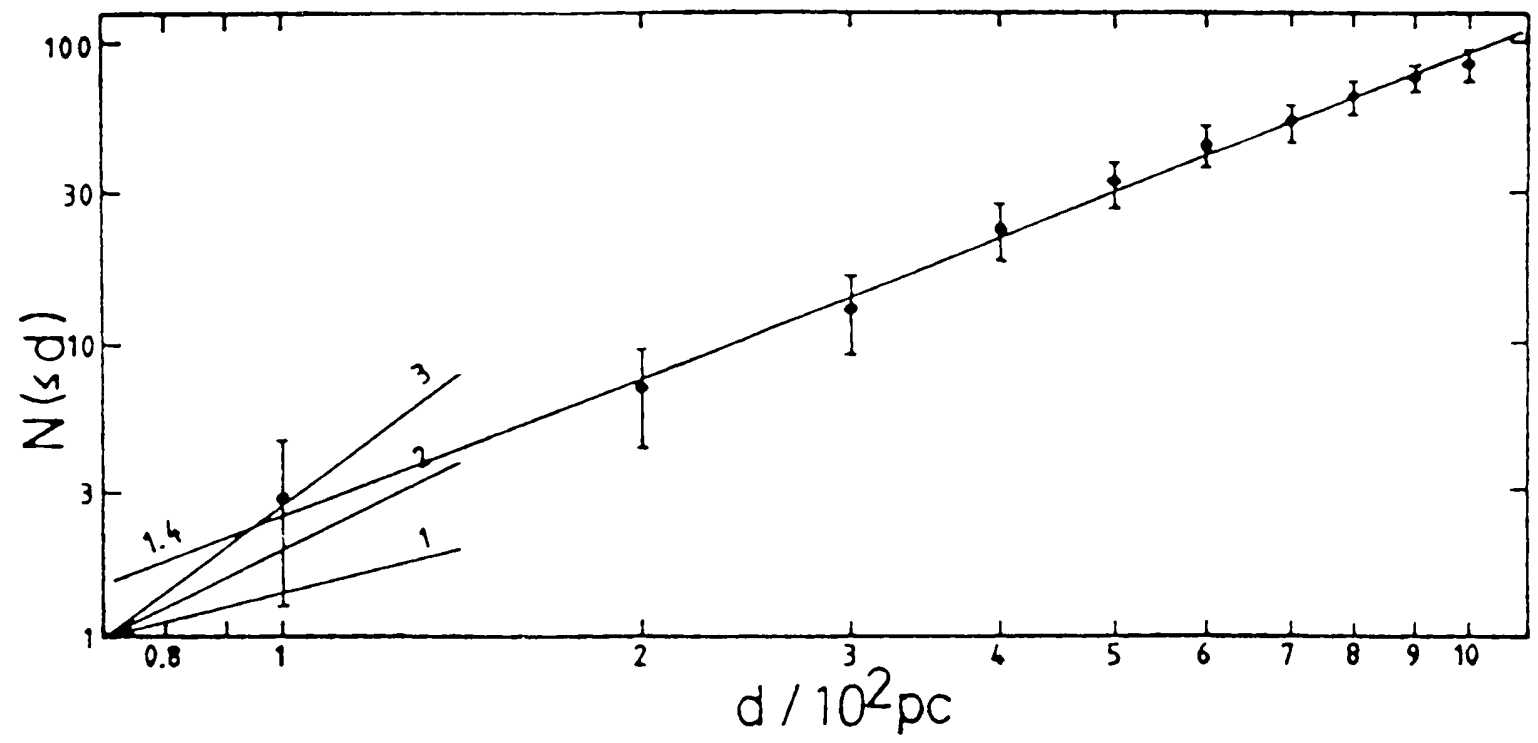

Figure 4 Number of pulsars $N$ at solar distances $\leq d$ versus $d$ for the 450 pulsars in Lyne and Smith (1990) and $d \leq 1 \mathrm{kpc}$. The slope of the observed power law is 1.4 , much smaller than the expected $\lesssim 3$, signalling incompleteness.

involve uncertain proper motions and scale heights at birth. As for $N$, the value in eq.(5) agrees with that in Davies et al. (1977), with a tendency of more recent reports to propose somewhat lower values. But figure 4 [whose data were calculated for the 450 pulsars in Lyne and Smith (1990)] shows impressively (by its low slope of the power law) that unless the Sun is at the center of a local pulsar concentration, the reported detections are incomplete even beyond $10^{2} \mathrm{pc}$ ! With $\geq 3$ pulsars within a volume of $10^{62} \mathrm{~cm}^{3}$, we have $\geq 10^{5.3}$ pulsars within the equivalent Galactic volume of $10^{68.8} \mathrm{~cm}^{3}$; this estimate even ignores a likely higher density along the $5 \mathrm{kpc}$ arm. I therefore favor an observable pulsar number in excess of $10^{5.3}$. An independent, local estimate of the beaming fraction $f$ has been obtained by Blaauw (1985): for $f=0.35$, a replenishment of the pulsar population within $0.5 \mathrm{kpc}$ of the Sun would require all stars born with $\geq 6 \mathrm{M}_{\odot}$ as progenitors. Again, neutron stars in binaries ask for progenitors of even lower masses, so that $f$ would be better of order unity.

This statistical consideration begs the question of whether pulsar beams are indeed pencil beams. If the radio pulses are emitted at a distance of $\gtrsim 1 \%$ of the speed-of-light radius above the polar capsas seems to be implied by the recent polarization data (Gil 1992) - the beam shapes should not differ a great deal from those of the polar caps. Are the latter circular? The polar caps are defined as the inverse images of the light cylinder under the map by the 'open' magnetic field lines, i.e. as the inverse images of infinitely long rectangles. They are expected to be elongated in latitude when the current-carrying corotating regions are taken properly into account. Curvature radiation is polarized with respect to the local curvature radii of the - not necessarily planar-field lines along the trumpet- shaped flux tubes. Any significant torsion of these field lines can render the proof by Lyne and Manchester (1988) inconclusive. Alternatively, emission at various heights from curved, circular flux tubes would likewise give rise to fan beams, cf. figure 1 .

A direct estimate of the fan beam elongation (of 5:1) has been attempted by Phillips (1990), based on the interpulse structure of PSR 1929+10.

If $f$ were much smaller than unity, i.e. if pulsars could be 'beamed away' from us, would not we expect to 'see' some of them through theirisotropically radiating - pulsar nebula? No convincing case is known to me (Seward and Wang 1988).

\section{Ages of the fastest pulsars}

The so-called millisecond pulsars are often thought to be spun up, or 'recycled' from slower pulsars. This interpretation was suggested by their occasional dim companions and by their abundance in globular clusters. However, recent proper-motion measurements have found kinetic ages of only a few $10^{5} \mathrm{yr}$ for 3 of them (Kulkarni 1990), just old enough for their SN shell to have faded away (Shull, Fesen, and Saken 1989). Moreover, spinup by accretion is not observed in the $\lesssim 30$ pulsing $\mathrm{X}$-ray sources, all of which show a balance between accretional spinup and magnetic spindown; a similar balance is expected for the non-pulsing sources. The millisecond pulsars may therefore be young and abundant, born faster because of a weaker coupling to their supernova shell (Kundt 1985, Kundt 1990b). They may turnoff young because of easier quenching.

Acknowledgments: I am particularly thankful for discussions with Jonathan Arons, Okie De Jager, Francis Graham Smith, Reinhold Schaaf, and John Seiradakis. 